\title{
POSSIBLE MECHANISMS FOR COMETARY OUTBURSTS
}

\author{
DAVID W. IIUGHES \\ Department of Physics \\ University of Sheffield \\ Sheffield, S3 7RH, U.K.
}

\begin{abstract}
A cometary outburst is an unexpected flare-up in the brightness of a comet, with the brightness normally increasing by 2 to 3 magnitudes, but on occasion by up to 5 . This outburst is superimposed on the general change in brightness that is related to the comet's heliocentric and geocentric distance, spin phase, spin axis orientation and activity geography. The brightness variation during outbursts usually has an abrupt, eruptive onset followed by a quasi-exponential decrease and lasts for a few tens of days. Several cometary outbursts are accompanied by the expansion of a spherical halo from the brightest point at the centre of the visible cometary coma.

The general characteristics of cometary outbursts and the suggested mechanisms put forward to explain their occurrences will be reviewed in this paper. (For a previous review of this subject see, for example, Hughes 1975.) We will only briefly touch on the 'superoutbursts' that occur when comets split apart. Special reference will be made as to the way in which the recent observations of the nucleus of $\mathrm{P} / \mathrm{Halley}$ have affected our views as to the possible causative mechanisms.
\end{abstract}

\section{The Characteristics of Outbursts}

There is little consensus as to what constitutes a typical cometary outburst, and the scientific literature is full of reports of the phenomenological differences between the different outbursts of different comets. This said, let us start by considering the outbursts of a comet that is famous for its ability to undergo many outbursts during its orbit.

P/Schwassmann-Wachmann I (1925 II, 1941 VI, 1957 IV and 1974 II), has a nearcircular orbit between Jupiter and Saturn, and during the comet's 15- to 16.4-year orbital period, the heliocentric distance varies from 5.45 to $7.46 \mathrm{AU}$. The orbital inclination is about 9.4 . With a large telescope the comet can be observed rather like an external planet, i.e., for about eight months of the year, when it is away from solar conjunction. Normally its photographic magnitude lies between 18 and 19. Schwassmann and Wachmann discovered it in 1927 (probably at the height of one of its outbursts), as a diffuse object of 13 to 14 magnitude. Soon observers were reporting that the comet underwent peculiar non-periodic variations in brightness. At relatively frequent intervals, the quiescent magnitude was enhanced by a sudden outburst that increased the brightness by a factor of 100 . The coma of the comet also increased its diameter, and a star-like condensation was much in evidence. 
Richter (1954) collected over 244 observations of the comet taken between 1939 and 1950. Twenty instances of outbursts were recorded. Whipple (1980) has recorded over 100 outburst flares for this comet over the previous fifty years. The expansion velocity of the edge of the inner coma region was of the order of 100 to $400 \mathrm{~m} \mathrm{sec}^{-1}$ (Richter 1954). A typical sequence of events is shown in Fig. 1. Initially there is a very faint diffuse coma, showing only a slight central condensation. Within a few hours, the central coma becomes star-like, and this is followed by a rapid increase in luminosity. This region then proceeds to expand until it resembles a planetary disc. More expansion changes the disc of uniform brightness into one with a brightness variation similar to the brightness of a cometary coma (i.e., $R^{-1}$ ). During the 'planetary disc stage', the spectrum is mainly that of scattered sunlight, but Cochran, Barker and Cochran (1980) have detected gaseous $\mathrm{CO}^{+}$. Note that the sequence of events is that which would be visible when using a small telescope. Note also that it is extremely difficult to get a spectrum at the height of an outburst. The dominance of the continuum probably only applies to the decay phase. Maximum luminosity is reached about 2.5 days after the onset of the outburst, at a time when the central cloud is about $300,000 \mathrm{~km}$ in radius. The material in this diffuse cloud is a poor reflector of light because the relative increase in the cross-sectional area of the cometary coma greatly exceeds the

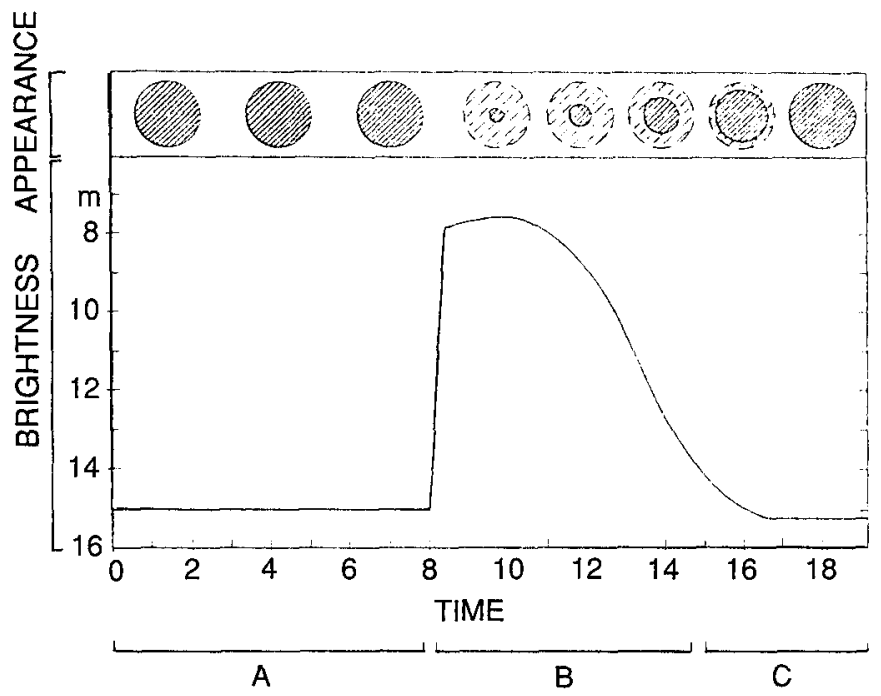

Fig. 1. The development of the physical appearance and the light curve of a cometary outburst, after Richter (1954). The sequence of events are those that would be visible through a small telescope. Stages $\mathrm{A}$ and $\mathrm{C}$ are similar and show the pre- and post-outburst comet. The spectrum at this stage is mainly a band spectrum produced by gas. Stage B shows the outburst phase where a star-like condensation is first seen in the centre of the coma. This condensation expands to become disc-like and then becomes fainter and finally disappears into the noise. The spectrum in phase B is mainly continuous and is caused by dust scattering. The numbers on the abscissa represent the time in days. The brightness is in magnitudes. 
observed increase in the luminosity. Roemer (1958) noted that the nebula disc had nearly faded from view when its diameter was between 2 and 3 arcmin. This usually occurred about 4 weeks after the onset, at a time when the radius was nearly $4 \times 10^{6} \mathrm{~km}$ (see also Roemer 1963, and Roemer and Lloyd 1966). Normally, there is very little coma activity between outbursts (see Degewij and Tedesco 1982), and Roemer (1958) described the coma's appearance as being not far from stellar. Jewitt (1990), however, observed the comet during the 1987-1988 perihelion passage phase, and found a steady and persistent low-level coma activity.

Cruikshank and Brown (1983) deduced from thermal emission measurements that the nucleus had a diameter of $40 \pm 5 \mathrm{~km}$.

Walker (1959) found that the radiation at the time of maximum brightness had a red excess $(B-V)$ of about +0.75 mag., this being typical of sunlight that has been scattered by dust particles of about $5 \times 10^{-5} \mathrm{~cm}$ diameter. According to Whitney (1955), the typical mass loss during an outburst of $\mathrm{P} /$ Schwassmann-Wachmann $\mathrm{I}$ is in the $10^{11}$ to $10^{12} \mathrm{~g}$ range, this mass having a kinetic energy of between $10^{19}$ and $5 \times 10^{19} \mathrm{erg}$.

No outburst has been seen to produce an observable effect on the orbital parameters of the comet, which indicates that the mass lost during the outburst is several orders of magnitude lower than the mass of the nucleus. Jewitt (1990) noted that the appearance of the outbursts depended considerably on the instrument, the detector, and the size of the effective diaphragm being used. Using a charge-coupled device (CCD) with a large telescope led to a different impression than that obtained when observing visually with a small instrument. The field of view and response nature differ considerably between these two systems.

Richter (1948a) looked for outbursts in other photometrically observed comets. Of the 358 comets seen since 1880 , he found that 12 had been observed to undergo outbursts. Richter concluded that "the phenomenological pattern of such brightness-outbursts is practically the same in all 12 cases, and furthermore that this is very similar to that observed for periodic comet Schwassmann-Wachmann I." Elizabeth Roemer (private communication) was not happy with this blanket statement and stresses that phenomenological differences were more noteworthy.

Richter (1948b) considered the role of cometary outbursts in the field of cometary discovery. In reviewing the lists of "unconfirmed comet discoveries" (20 objects appeared on this list in the interval 1880 to 1930 ), he stressed that the first sighting could easily have occurred at the time of outburst. After the subsequent rapid decline in brightness, the comet quickly became lost from view. A typical, but less catastrophic, incident occurred with comet Pajdusakova (1954 II). This was discovered on 1953 December 3 when it was at magnitude 11. It was expected to brighten as it moved towards perihelion $(0.07 \mathrm{AU})$ on 1954 January 24 . Instead it faded to $14 \mathrm{mag}$. by January 10 . Maybe it was discovered at outburst. Kresák (1966) noted that P/Faye in 1843, P/Brooks 2 in 1889, P/Holmes in 1892 and P/Grigg-Skjellerup in 1909 also fell in this category and that the brightness at discovery often biased serious attempts to chart the secular decrease in cometary activity. Pittich (1969) found that, during the last two centuries, at least 40 comets appeared to have suddenly increased in brightness shortly before their discovery. This conclusion came from an analysis of the time intervals over which the comets remained undiscovered in spite of favourable observing conditions. A list of these comets is given in Pittich (1971). This paper also contains a list of all the comets that have been seen to outburst. 
The two unprecedentedly violent outbursts of P/Tuttle-Giacobini-Kresák (1858 III, 1907 III, $1951 \mathrm{IV}, 1962 \mathrm{~V}, 1973 \mathrm{VI}$ and $1978 \mathrm{XXV}$ ) that took place around 1973 May 28 and 1973 July 7 are worthy of note (see Fig. 2). Kresák (1974) reported that the comet had been observed to be rather inactive, faint and diffuse until that time. Unfortunately the comet-Sun-Earth geometry at previous apparitions was such that similar outbursts in the past had an $80 \%$ chance of not being noticed. In the past the comet had an absolute magnitude of 11.3 (1858), 12.3 (1907), 12.0 (1951) and 11.3 (1962), indicating that the nucleus is small, the radius being about $0.3 \mathrm{~km}$ according to the formula given in Hughes (1988a).

The first outburst began on 1973 May 25.0, and the brightness increased at the rate of 3 magnitudes per day until maximum was reached on May 28.0. At that time, the brightness had increased by a factor of 4000 , equivalent to a cliange in magnitude of 9 . The decay occurred at a rate of about $1.2 \mathrm{mag} \mathrm{day}^{-1}$. The second outburst began on July 5.0 , and an increase of 9 magnitudes was again achieved, but this time in about two days. At the maximum, the coma was very nearly circular and had a radius of $55,000 \mathrm{~km}$. A very faint halo surrounded this inner region and extended out to a radius of $85,000 \mathrm{~km}$. This halo blended into a faint, featureless tail which extended $600,000 \mathrm{~km}$ in the anti-solar direction. At maximum the coma was 100,000 times brighter than the nucleus. The expansion velocity was somewhat in excess of $500 \mathrm{~m} \mathrm{sec}^{-1}$. (Jewitt 1990 found that the expansion velocity of the outburst coma of $\mathrm{P} / \mathrm{Sch}$ wassmann Wachmann I was about $200 \mathrm{~m} \mathrm{sec}^{-1}$.) One day after maximum, three distinct regions could be seen in the coma, with the inner two having radii of 8,000 and $35,000 \mathrm{~km}$. The outer halo had become elongated in the direction perpendicular to the tail direction. A bright jet protruded from the nucleus and pointed in the anti-solar direction. Material in the jet had a velocity of at least $700 \mathrm{~m} \mathrm{sec}^{-1}$. Spectra indicated that the jet was composed of escaping dust, whereas the inner coma was mainly CN.

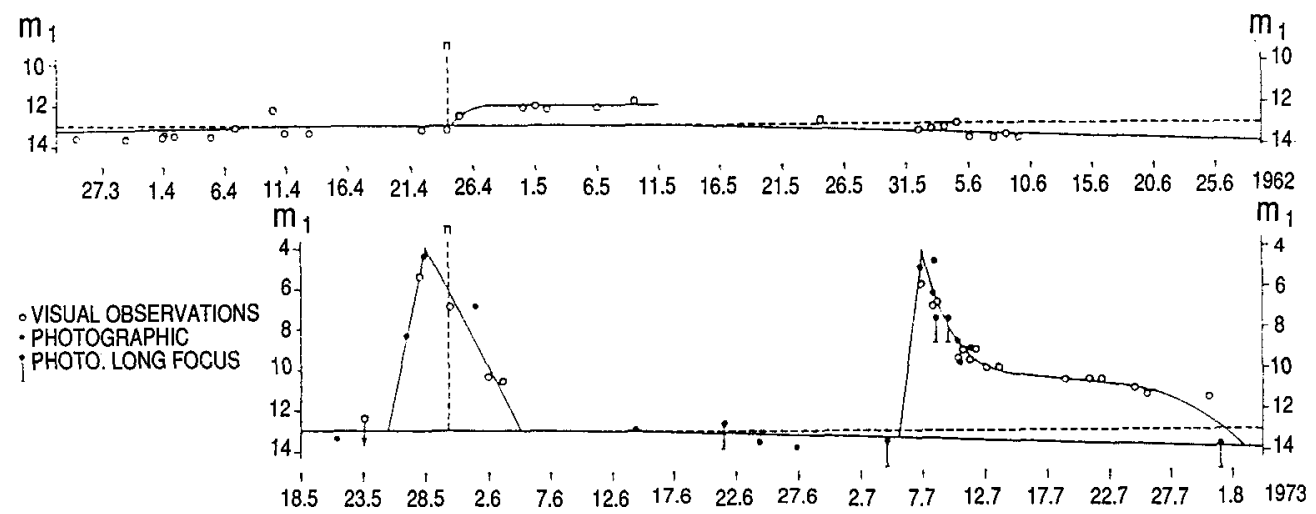

Fig. 2. The light-curves of comet P/Tuttle-Giacobini-Kresák (after Kresák 1974). The upper curve shows the appearance in 1962 (the comet was $1962 \mathrm{~V}$ ). The lower curve shows the two cometary outbursts that took place in May and July 1973 (the comet was 1973 VI). The vertical dashed line represents the perihelion passage. The ordinate represents the heliocentric magnitude, i.e., the apparent magnitude minus $5 \log \Delta$, where $\Delta \mathrm{AU}$ is the comet-Earth distance. 
Kresák found that the total energy utilised by the comet during the two outbursts (i.e., within a few days) was equal to that required for the activity of 80 normal orbits. He also noted that the occurrence of two outbursts in a short time span by the same comet was not uncommon.

The list given by Pittich (1971) shows that outbursts are certainly not confined to the short-period comets. Long-period comets Morehouse (1908 III) and Humason (1962 VIII) are noteworthy. The latter had a "crab-like" appearance as it approached the Sun (see Roemer 1962, and Brandt and Mendis 1979). It remained active during the whole of its apparition and underwent a 6-magnitude outburst in 1964 when it was 6 AU from the Sun.


Fig. 3. (a) The symbols represent the positions of comets at the time of observed outbursts (after Pittich 1972). The abscissa is the ecliptic heliocentric distance in AU, and the ordinate is the distance perpendicular to the ecliptic plane (in AU). The dots are the observed cometary outbursts, the circles are the hypothetical outbursts that probably preceded the discovery of certain comets, and the triangles are the outbursts of P/Schwassmann-Wachmann I. (b) The data of Fig. 3a have been sorted into 0.25-AU bins, and the number of outbursts occurring in each region ( $r$ to $r+0.25 \mathrm{AU})$ is plotted as a function of heliocentric distance. 
Another interesting clue as to the possible mechanism responsible for the outbursts comes from an analysis of the positions of the comets in the solar system at the time of the outburst (see Fig. 3a). There is a distinct asymmetry with respect to the ecliptic plane, with two-thirds of the observed outbursts occurring north of the ecliptic. This is a selection effect reflecting the geographical distribution of the observers.

The median declination of $\mathrm{P} /$ Schwassmann-Wachmann $\mathrm{I}$ at the time of outburst was $+18^{\circ}$. For all the other comets, this value was $+25^{\circ}$.

It is interesting to note that the median declination of comets at the time when they split is $+1^{\circ}$ and splitting seems to be much more concentrated towards the celestial ecliptic. Selection effects could play a part in this observed concentration. Most short-period comets have low inclinations and thus do not stray far from the ecliptic. Also most comets are observed when they are close to the Sun, which is on the ecliptic.

Hughes, Searle and Street (1974) analysed the spatial distribution of cometary outbursts (see Fig. 3b). Three regions were apparent:

(a) for heliocentric distance $r<1 \mathrm{AU}$, the distribution simply reflected the distribution of cometary perihelion distances.

(b) for $1.0<r<5.5 \mathrm{AU}$, the distribution followed an $r^{-2.2}$ law, this probably being a result of observational selection as the ratio between the number of detected and undetected outbursts decreased as the Earth-comet distance increased. This power law is similar to the law describing the observed (as opposed to actual) perihelion distribution of comets. If outbursts occurred at set of specific heliocentric distances, corresponding to a series of snow sublimation temperatures, the outburst frequencies would be bunched around these $r$ values and would not simply follow the observed distribution of perihelion distances.

(c) for $r>5.5 \mathrm{AU}$, the data originates in toto from the analysis of two complete orbits of P/Schwassmann-Wachmann I (see Patashnick, Rupprecht and Schuerman 1974). No clear variation in the number of outbursts with heliocentric distance is seen.

A tentative conclusion is that the outburst probability is independent of heliocentric distance, at least for $r<7.5 \mathrm{AU}$. This means that this probability is similarly independent of the surface temperature of the cometary nticleus. The temperature of the subsolar point of a cometary nucleus composed completely of water snow, according to Delsemme (1966), is given as

$\begin{array}{lllllllll}r(\mathrm{AU}) & 6.93 & 6.18 & 5.10 & 3.70 & 2.18 & 1.13 & 0.60 & 0.30 \\ T(\mathrm{~K}) & 150 & 160 & 170 & 180 & 190 & 200 & 210 & 200\end{array}$

(see also Delsemme 1985). The surface of a thick, low-albedo dust layer on the nucleus would be much higher and would probably be close to the Zanstra temperature $\left(290 r^{-0.5} \mathrm{~K}\right)$.

Miller (1961), and also Campins and Ferrin (1978), noted that cometary outbursts occurred more often after perihelion than before (see Fig. 4). This is the time when the heat content of the nucleus reaches its maximum and when periodic comets usually have their highest activity. It seems reasonable to conclude from this observation that outbursts are more prevalent at times of high cometary activity. It must be said, though, that many cometary discoveries seem to occur when the comet is close to perihelion, and then the comet is followed for a long time after perihelion. So for new (Oort-Öpik cloud) comets, 
there are more post- as opposed to pre-perihelion observation and thus one would expect to observe more post-perihelion outbursts than pre-perihelion ones. This is not the case for periodic comets. These have a more symmetrical observation period with respect to perihelion passage.

Delsemme (1986) concluded that both long-period comets (i.e., comets that have not decayed significantly since their time of origin) and short-period comets (comets that have had many close passages of the Sun and have lost considerable amounts of mass) appear to split at the same rate, with there being about a $3 \%$ chance per perihelion passage. This indicates that, with the exception of the surface dust layer, the overall structural strength of the cometary material and its bulk chemistry do not change significantly as a function of position inside the nucleus. In terms of outburst statistics, we can conclude that comets have about the same chance of undergoing outbursts at any stage in their evolution, and that the propensity for outbursts is not a function of the size of the cometary nucleus, the time the comet has spent in the inner solar system, or the orbital parameters.

Outbursts of P/Halley have been reported by Larson et al. (1989) and Feldman et al. $(1986 \mathrm{a}$ and $\mathrm{b})$. It seems that low $\Delta m$ outbursts were hard to detect and were usually overlooked. They tended to be hidden by the error noise inherent in the magnitude-estimating process. Care also had to be taken not to confuse the short-term variations in the brightness caused by the rotation of the nucleus, with small outbursts. The spectrum at outburst time was mainly of the reflection type, this probably being caused by an enhancement of dust and 'dirty' ice grains in the vicinity of the nucleus. The gas spectrum at outburst was less prominent but was certainly evident when being specifically looked for. Larson et al. (1989) were monitoring the $\mathrm{H}_{2} \mathrm{O}$ production of $\mathrm{P} / \mathrm{Halley}$ using a Fourier transform spectrometer on board the Kuiper Airborne Observatory. On UT 1986 March 20.7 they recorded an outburst that showed the $\mathrm{H}_{2} 0$ brightness increase by a factor of 2.2 in less than $10 \mathrm{~min}$.

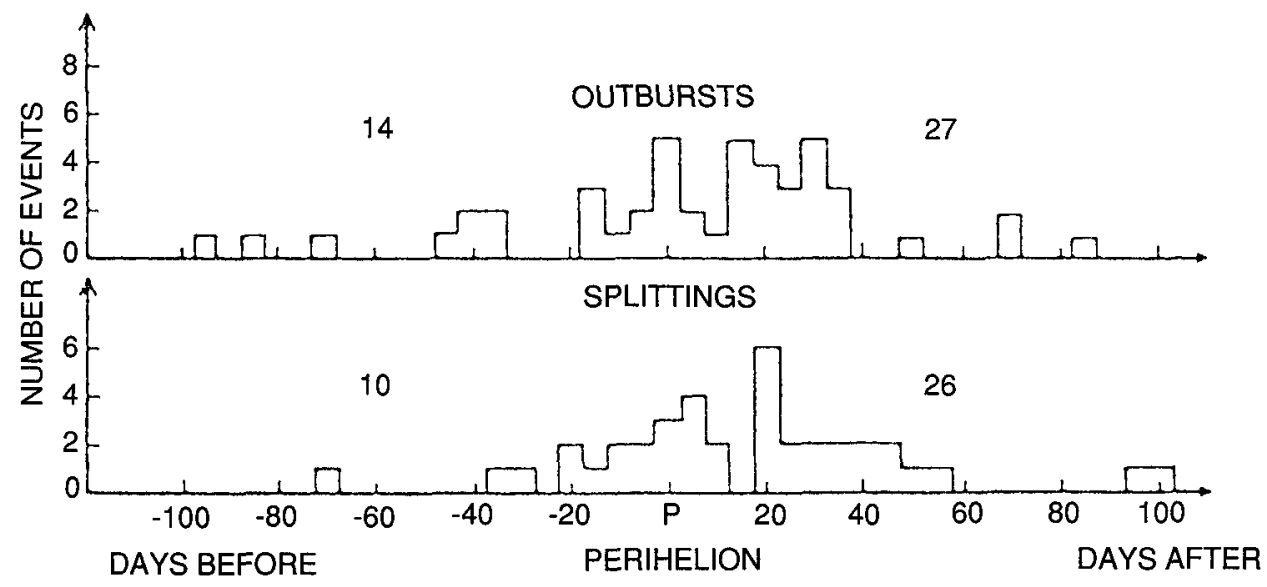

Fig. 4. The observed numbers of outbursts and cometary splittings are plotted as a function of time with respect to the perihelion passage of the comet (after Miller 1961). Data from 87 comets are included. 
Flammer et al. (1986) showed that many of P/Halley's small outbursts at large heliocentric distances could be temporally correlated with the passage of a high-speed solar wind stream. The authors suggest that fine, electrically-charged dust was being elevated from the surface of the nucleus by electrostatic forces. The increase in the surface area exposed to sunlight accounted for the outburst. Probably the large majority of this dust returned to the nucleus when the density and speed of the wind dropped.

Feldman et al. (1986a) used the Fine Error Sensor on the International Ultraviolet Explorer (IUE) spacecraft as a photometric monitor of the activity of $\mathrm{P} / \mathrm{Halley}$. This sensor has a field of view of $16 \times 16$ arcsec. The authors noted that large amplitude variations in brightness, in the broad wavelength band $4000-6500 \AA$, were particularly noticeable during the last week of December $1985(r \simeq 1.07 \mathrm{AU})$ and during March and April $1986(0.71<r<1.62 \mathrm{AU})$. These variations were attributed to a combination of two things: first, the effect of the rotation of the nucleus, and second, the outbursts of individual jets from active areas on the nucleus surface. The two outbursts in the last week of December showed a maximum to minimum brightness ratio of 2.0 and 2.5 , equivalent to magnitude changes of 0.75 and 1.0, respectively. A sharp outburst on March 18-19 correlated with the observation by the IUE of both enhanced $\mathrm{CO}_{2}^{+} 150,000 \mathrm{~km}$ tailward of the nucleus and of a large detached cloud of $\mathrm{CO}^{+}$on March 20 (see Feldman et al. $1986 \mathrm{~b}$ ). The magnitude changes in March were of the order of 1.0. (Note that these are similar to the changes in magnitude $(\Delta m=0.8)$ reported for the spin modulation.) These observations could point to a certain degree of compositoinal inhomogeneity in the cometary snows. Other outbursts, without the carbon compound features, were referred to as $\mathrm{H}_{2} \mathrm{O}$ outbursts, and these were of a shorter time-scale.

How does the number of observed outbursts vary as a function of the magnitude of the outburst? A crude analysis of the P/Schwassmann-Wachmann I data given in Richter (1941) is shown in Figure 5. The scarcity of $\Delta m=1$ outbursts is probably due to these small outbursts being lost in both the magnitude measurement noise and the spin-induced magnitude modulation. Two distributions are suggested by Fig. 5:

(i) outbursts cluster about a mean $\Delta m$ of about 2.5 .

(ii) outbursts have a whole range of $\Delta m$ values, the outbursts being less frequent as $\Delta m$ gets larger. The latter is the most probable.

Whitney (1955) noted that the outbursts of comets Holmes, Swift and Pons-Brooks were relatively more violent than those of $\mathrm{P} / \mathrm{Sch}$ wassmann-Wachmann I. These comets have smaller perihelion distances and thus the potential to activate more parent molecules. They also have much larger eccentricities. Little $\mathrm{H}_{2} \mathrm{O}$ sublimation is expected in the $5.5<$ $r<7.5 \mathrm{AU}$ region of the solar system in which P/Schwassmann-Wachmann I orbits.

IIow many of the observed comets undergo outbursts? Unfortunately answers vary. Vsekhsviatsky (1966) found that 59 out of a sample of 79 comets that passed perihelion between 1935 and 1975 underwent outbursts. Taking into account sky conditions and periods when observations were not available, he concluded that "all comets can experience brightness variations." Richter (1963) noted that some comets were much more prone to outbursts than others, but concluded that it was impossible to decide whether these were specific types of comets or comets at a certain stage of their evolution. Kresák (1974) was more positive and stated that both comets that had just been captured into short-period orbits, and comets that had ablated to a small nuclear core and were approaching their final disruption were more likely to outburst than comets at more stable phases of their 
evolution. Outbursts, like splittings, have been associated with high spin rates.

The relationship between outburst and solar activity is somewhat tenuous. Richter (1954) found that the outbursts of P/Schwassmann-Wachmann I were temporally correlated with the occurrence of solar M-regions. Whitney (1955), however, pointed out that the amount of energy that could be absorbed from the impinging solar wind was insufficient to supply the energy needed for the outburst and so the wind could only act as a trigger. Vsekhsviatsky (1966) studied the decay phase of the outbursts of P/SchwassmannWachmann I and noted that there were slight increases in the brightness in the decay profile around the periods 25-27 and 50-60 days after the outburst (see Fig. 6a). He took this to indicate that the outburst was triggered by emissions from a solar active region and that these emissions also affected the expanding cometary cloud of gas and dust each time the solar rotation brought the region into view.

Vsekhsviatsky (1966) also plotted the number of observed cometary outbursts as a function of solar activity (see Fig. $6 \mathrm{~b}$ ). IIe concluded that the outburst rate maximised at
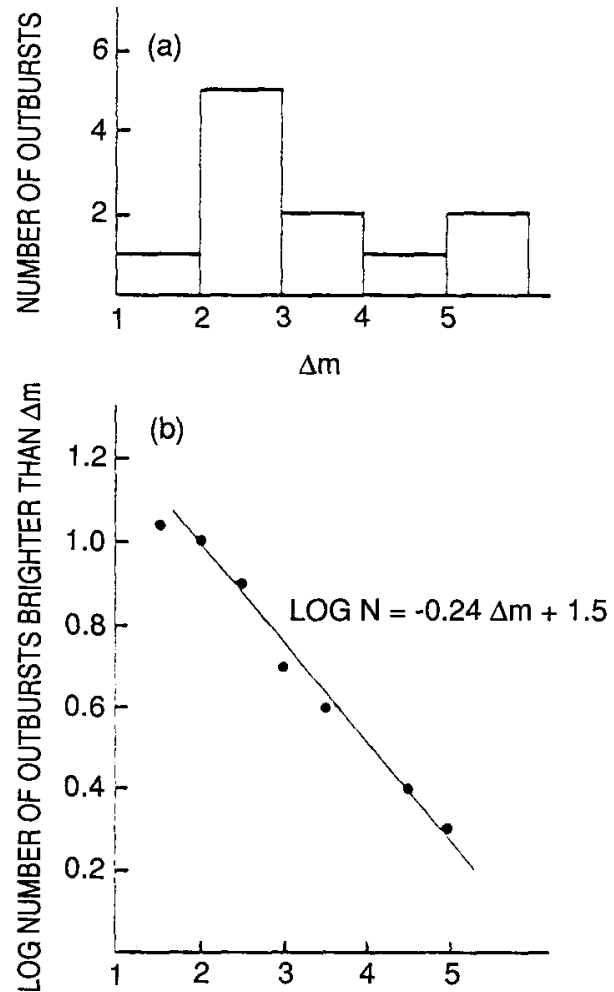

Fig. 5. The number of observed outbursts of comet $P /$ Schwassmann-Wachmann $I$ is plotted as a function of the brightness of the outburst. The data have been taken from Richter (1941). (a) shows a histogram representation of the data; (b) shows the logarithm of the number of outbursts brighter than $\Delta m$ plotted as a function of $\Delta m$. 
both the 0.5-0.6 phase and the 0.8 phase of the cycle (where 0 and 1.0 are successive sunspot activity minima). There is, unfortunately, a large statistical variation in the data, and Kresák (1974) was not convinced as to the significance of the correlation. If outbursts were triggered by solar activity, Kresák noted that it was difficult to explain why intense solar flares did not always produce cometary outbursts and why all the comets that happened to be close to the Sun at the time of the flare did not respond to its influence in a similar way. The two violent outbursts of $\mathrm{P} /$ Tuttle-Giacobini-Kresák in 1973 were separated in time by 40 days and the outbursts had no temporal correlation with any indicator of solar activity.

The characteristics of cometary outbursts are summarised in Table 1.
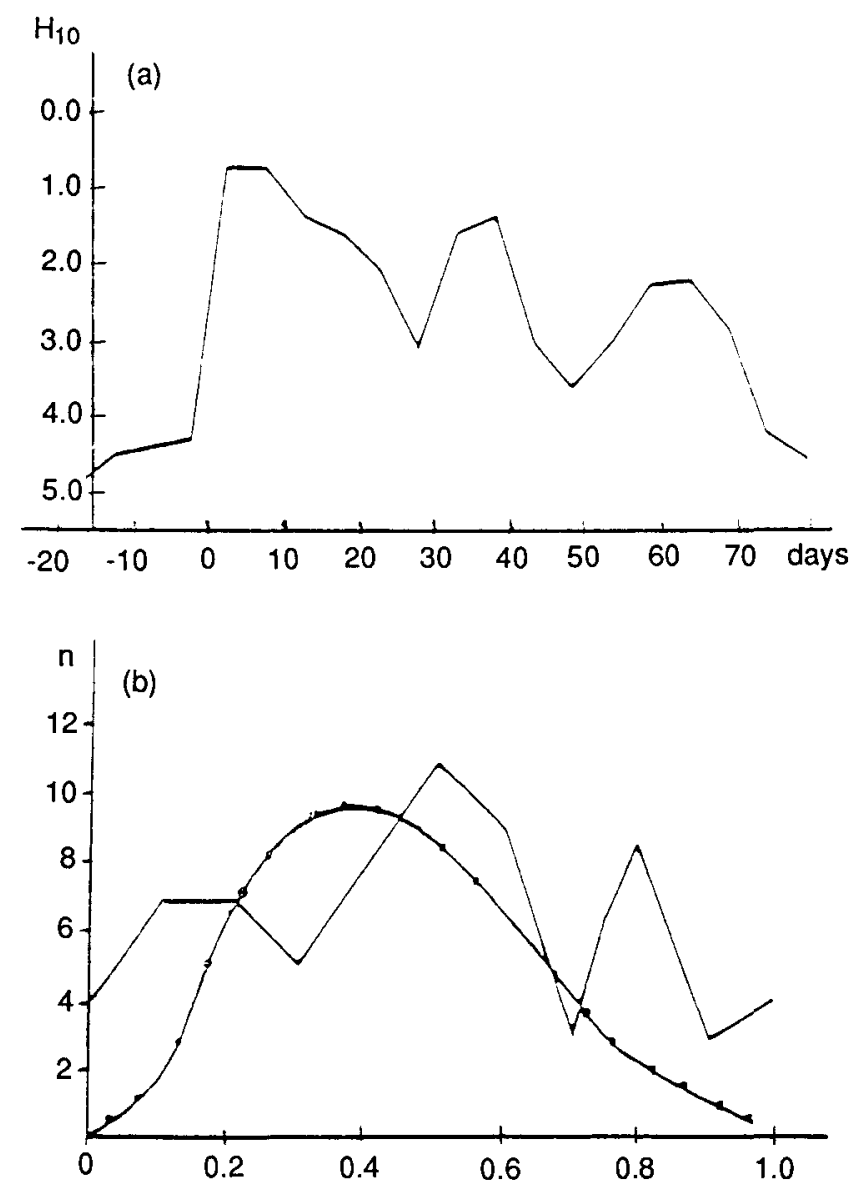

Fig. 6. (a) The brightness of P/Schwassmann-Wachmann I during an outburst. The decay profile shows a strong 26-day periodicity, a periodicity that is close to the solar rotation period (see Vsekhsvyatskii 1966). (b) The time of occurrence of cometary outbursts is plotted as a function of the solar sunspot activity phase. Successive sunspot minima are represented by 0.0 and 1.0 (see Vsekhsvyatskii 1966). 
Table 1. The Characteristics of Comctary Outbursts

1. There is a sudden, sharp increase in brightness, and the typical magnitude change is about 2 , but values ane found in the range of 1 to 5 .

2. The brightuess increase takes around 2.5 days, and the decay about 20 to 30 days.

3. The comet relums to normal after the outburst.

4. The spectrum of the outburst is mainly due to dust scittering, with a B-V of about 0.75 , indicating that the effective dust particles are around $10^{-4} \mathrm{~cm}$ in size.

5. The expansion velocity of the outburst cloud is in the range of $10010400 \mathrm{~m} \mathrm{sec}^{-1}$, and the maximum brightness occurs when the cloud has a diameter of about $300,000 \mathrm{~km}$.

6. The shape of the cloud can be circular, oval or asymmetric.

7. The mass loss from the conce during a typical outburst is in the range of $10^{11}$ to $10^{12} \mathrm{~g}$, cepuivalem to the loss of about $50 \mathrm{~km}^{3}$ of malcrial.

8. The comclary gas production increases by around $10^{3} 1010^{4}$.

9. No outburst has been seen to result in a change in the comel's orbit.

10. The occurrence of outbursts scems to be random in the inner solar system, so the mechanism responsible is not a function of the surfice temperature of the nucleus.

11. The occurrence rate is higher post-perilhelion, indicating that the nechanism is a function of cometary activity.

12. There is a Icndency for outbursts on occur in pairs, with this activity being followed by long intervals of quict crolution.

13. Many conets, of bolh shom and long period, have been observed to ouburst, and it seems reasonable to suppose that all comes can and do sulfer from outbursts.

\section{Possible Outburst Production Mechanisms}

A cometary nucleus changes as a function of time (see Hughes 1985 and 1987). P/Halley (see Feldman et al. 1986a) lost $1.5 \times 10^{14} \mathrm{~g}$ of $\mathrm{H}_{2} \mathrm{O}$ during the time interval September 12,1985 , to July 8,1986 , i.e., when $\mathrm{P} /$ Halley was closer to the Sun than $2.6 \mathrm{AU}$. At the time of the Giotto flyby, the comet was losing dust at the rate of $3.3 \times 10^{6} \mathrm{~g} \mathrm{sec}^{-1}$ (see McDonnell et al. 1986). The total gas emission rate at that time was $2.7 \times 10^{7} \mathrm{~g}$ $\mathrm{sec}^{-1}$ (Krankowsky et al. 1986), giving a dust-to-gas mass ratio of $1 / 6$. Unfortunately, the Giotto data gave no details of the amount of dust present in the form of particles with masses greater than $1 \mathrm{~g}$ (i.e., radii greater than $1 \mathrm{~cm}$ ). McDonnell et al. (1989) modelled the effect of including these larger dust particles and found that no only was the lower limit for the dust-to-gas mass ratio 1.0, but that it could possibly be of the order of 200 . The latter value is obtained if the largest particle that can be blown away from the nucleus by gas pressure is assumed to be about $25 \mathrm{~cm}$ in radius (i.e., has a mass of $20 \mathrm{~kg}$ ).

So we have a dilemma. The mass of the $\mathrm{P} / \mathrm{Halley}$ nucleus has been estimated to be of 
the order of $1.5 \times 10^{17} \mathrm{~g}$. This value comes from multiplying the volume obtained from the measured surface area of $400 \mathrm{~km}^{2}$ (Keller et al. 1986) by an assumed density of $0.2 \mathrm{~g} \mathrm{~cm}^{-3}$ (Rickman 1986). The total gas loss by the comet during the perihelion passage will be slightly greater than the $\mathrm{H}_{2} \mathrm{O}$ value given above and can be taken to be $1.6 \times 10^{14} \mathrm{~g}$. The total mass loss by the comet will be $(1+d)$ times this value, where $d$ is the dust-to-gas mass ratio. For $d$ values of $0.17,1.0,10.0$ and 200 , the mass loss during the recent apparition of the comet is $1.9 \times 10^{14}, 3.2 \times 10^{14}, 1.8 \times 10^{16}$ and $3.2 \times 10^{16} \mathrm{~g}$. These values are equivalent to the loss of a complete surface layer of the nucleus of thickness $2.3,4,22$ and $400 \mathrm{~m}$, respectively. If the nucleus remains active over only $10 \%$ of its surface, the layer loss rate will be ten times larger in the active region than the values given above. If the middle to higher values of $d$ are the correct ones, it is clear that the surface is changing quite dramatically as a function of time during the comet's sojourn in the inner solar system. The Giotto camera revealed that the surface of the nucleus of P/Halley was smooth on a scale of a few hundred metres. This indicates that the active regions are not fixed in position and that the variation in the activity geography from apparition to apparition tends to round off the topography.

The decay of comets depends strongly on their perihelion distances, $q$. Hughes (1986b) concluded that the mass loss is proportional to $q^{-(m-1.5)}$, where $m$ is in the range 2 to 3 . So a change in perihelion distance from 0.1 to $1.7 \mathrm{AU}$ decreases the decay rate by a factor of 4 or 70 for values of $m$ equal to 2 and 3, respectively. Comets with perihelia in the more distant reaches of the solar system have surfaces that change much more slowly than that of $\mathrm{P} / \mathrm{Hall}$ ey. We must remember, however, that the prevalence of outbursts does not seem to be a function of heliocentric distance. Also, comets could have a range of dust and snow compositions and a range of dust-to-gas mass ratios. These could be a function of the heliocentric distance at which the comet formed. This diversity could also apply to the planetesimals that came together to form each individual comet.

We will now list, in no specific order, the mechanisms that have been put forward as possible causes of cometary outbursts. Most rely on some agent leading to the violent removal from the nucleus of a considerable amount of cometary material. This has two obvious consequences.

First, the percentage of the nucleus that is actively emitting gas and dust can be increased dramatically. Note that Hughes (1988b) concluded that, over the last 2000 years, $\mathrm{P} /$ Halley was, on the average, active over only $3.4 \%$ of its surface. The percentage of the surface that was active varied widely from apparition to apparition. The lowest value was $0.8 \%$ and the highest was $10 \%$. These values could be typical of comets in general. If, say, the outburst temporarily changed the active percentage from $0.8 \%$ to $40 \%$, this factor of 50 is equivalent to a change in cometary magnitude of 4.25 .

Second, a violent crustal removal process could lead to the fragmentation of the emitted dust particles and a consequent increase in the mass distribution index of the dust and the surface-area-to-mass ratio of the emitted material (see, for example, Figure 1 in Daniels and Hughes 1981). So mass for mass, the dust emitted after an outburst could be a more effective scatterer of sunlight than the dust that is normally emitted from the comet. (Note that if the emitted particles were broken up into fragments were that smaller than, say, $0.1 \mu \mathrm{m}$, a particle size that does not scatter light very efficiently, this breakup would result in less light being scattered.) 


\subsection{Pressure Released From Gas Pockets}

Whitney (1955) postulated that the cometary vapours released by sublimating snows could become concentrated just below the surface of the nucleus. The resultant pressure build-up might act as a centre of stress and the subsequent explosive release of this high pressure could remove considerable portions of the crust and expose a large region of the underlying snow to the action of sunlight. Minor species such as $\mathrm{CH}_{4}, \mathrm{CO}$, or $\mathrm{CO}_{2}$ could be the propellant gas. The sublimation rate of all species is temperature-dependent, and the species mentioned above all sublimate at temperatures well below those associated with the sublimation of $\mathrm{H}_{2} \mathrm{O}$, the most common cometary constiuent. $\mathrm{CH}_{4}$, for example, is very volatile and produces a considerable vapour pressure at temperatures as low as $40 \mathrm{~K}$. Strong sublimation will be taking place at a temperature of around $130 \mathrm{~K}$, a value that can be attained at the surface of a low-albedo, slowly rotating comet at around $6 \mathrm{AU}$ on the inward journey.

This hypothesis runs into certain problems. Unfortunately, this mechanism is temperature dependent in a rather odd way in that the resultant outbursts would be clustered at those heliocentric distances that corresponded with the onset of the massive sublimation of specific species. The suggested mechanism would be less effective when the cometary temperatures are decreasing, i.e., on the outward leg of the orbit, a conclusion that contradicts the findings shown in Fig. 4. It is also possible that the individual species would produce outbursts that were phenomenologically different, with the minor species leading to smaller outbursts than the more major ones. So the size of the outburst would depend on the heliocentric distance. None of these attributes are seen in the outburst data. There is a possibility that $\mathrm{P} / \mathrm{Schwassmann}$-Wachmann I would be too cold at the aphelion of its orbit for this mechanism to be effective, although this statement does depend on the assumed chemistry of the cometary snows.

The strongest objection to this mechanism is based on our paradigm of the structure of the cometary surface and interior. Measurements of the strength and density of meteoroids (millimetre to centimetre sized ejected fragments of cometary dust crusts - i.e., cometary material that has lost the interstitial snows) lead to the conclusion that cometary dust has a very low density and is porous, crumbly, vesicular and fragile (see Hughes 1978 and Greenberg 1986). The low densities that have been found for the cometary nuclei (see Rickman 1986, Rickman et al. 1987, Smoluchowski 1986, Wallis and McPherson 1981 and Whipple 1987) indicate that these attributes also apply to the nucleus in general. The nucleus is like a sponge and is thus very unlikely to support the build-up of gas pressure. The porosity of the nucleus has been stressed by Smoluchowski (1985) and Iloranyi et al. (1984). The only way in which a model sponge nucleus can be made impermeable to gas is to reduce the pore size of the sponge down to the order of $10^{-4} \mathrm{~cm}$ (Jacklyn Green, private communication, 1989). Here the extremely tortuous path that the gas must follow in order to escape leads to many dead ends and a build-up of pressure. It must be noted that some researchers do not agree with the low-density model of the nucleus and Yeomans (1989) and Peale (1989) both suggest that the density may be nearer to $1 \mathrm{~g} \mathrm{~cm}^{-3}$. 


\subsection{Explosive Radicals}

Donn and Urey (1956) suggested that some of the constituents of the cometary snows could be transformed chemically into explosive mixtures under certain conditions. The free radical $\mathrm{NH}$ is reasonably stable at low temperatures, but, on being heated in a cometary snow environment, would be converted to the explosive ammonium azide $\left(\mathrm{NH}_{4} \mathrm{~N}_{3}\right)$ at a temperature of $148 \mathrm{~K}$. The central temperature of $\mathrm{P} / \mathrm{Halley}$ is of the order of $70 \mathrm{~K}$ (see Hermann and Weissman 1987). Near perihelion, the $148 \mathrm{~K}$ region would be a few tens of metres below the surface.

Another explosive radical is $\mathrm{OH}$, which combines to form hydrogen peroxide at $77 \mathrm{~K}$. Mixtures of $\mathrm{H}_{2} \mathrm{O}_{2}$ with carbon compounds are highly explosive. Dilution with stable compounds leads to an inert nucleus, but the progressive sublimation of these dilutants might produce a 'space bomb' which could be easily detonated by a localised source of energy.

Unfortunately, none of the present-day cometary models predict the existence of these species. Even if the mantles of pre-cometary dust particles contained them, the accretion process would have led to their thermal destruction. The 'explosives' have parent molecules that are very minor constituents, and there is every expectation that these are well-mixed with the more common cometary snows. An outburst of 2 to 3 magnitudes would require a considerable explosive charge, and there seems to be no efficient mechanism that could lead to the concentration of the explosives. (Note that a process in which $\mathrm{CO}_{2}$ sublimates near the surface, but then, in the main, diffuses into the comet to condense in cooler regions, has been suggested by Smoluchowski 1985 as a possible way of increasing the concentration of this species.)

Returning to the explosive radicals, it is probable that the continuous sublimation of the cometary snows would tend to remove them nearly as quickly as they formed. This could lead to a series of very minor explosions, but these would be lost in the general noise of the everyday variation of cometary brightness. The mechanism is also strongly temperature-dependent and would probably, like the mechanism described in section 2.1, only lead to the production of 'explosive' outbursts when the temperature gradient was positive, i.e., when the comet was on the inward path of its trajectory.

A chemical explosion could produce a visible short-duration flash at the onset of the outburst. These flashes have not been observed, but unfortunately the chances of seeing one are exceedingly small, so this not a useful argument against the mechanism.

\subsection{Amorphous Ice}

The Giotto gas mass spectrometer found that around 16 out of every 20 molecules emitted from $\mathrm{P} / \mathrm{Halley}$ were $\mathrm{H}_{2} \mathrm{O}$. Comets were probably formed by planetesimal accretion in the Saturn to Neptune region or beyond. The solidification of water vapour at temperatures of less than $95 \mathrm{~K}$ and at pressures of less than $1 \mathrm{~N} \mathrm{~m}^{-2}$ could form high-density amorphous ice and not the everyday low-density hexagonal variety. (I use the word 'could' because the effect of large amounts of dust in the vicinity might significantly change the end product.) Patashnik et al. (1974) postulated that cometary outbursts were simply produced when the amorphous ice in the nucleus surface region underwent a phase change to cubic ice at a temperature near $140 \mathrm{~K}$ (see also Klinger 1980 and Smoluchowski 1981). This transition releases considerable energy (about $9 \times 10^{8} \mathrm{erg} \mathrm{g}^{-1}$ ). 
Amorphous ice is denser than cubic ice, and Patashnik et al. used a value of $2.3 \mathrm{~g} \mathrm{~cm}^{-3}$ (see Delsemme and Wenger 1970), although a more typical value is $1.2 \mathrm{~g} \mathrm{~cm}^{-3}$ (see Venkatesh et al. 1974). Cubic ice has a density of $0.94 \mathrm{~g} \mathrm{~cm}^{-3}$. The rapid expansion of the ice produced by the phase change causes the pulverisation of the surrounding regions of the nucleus. The transition region would be the 'warm' snow just under the dust crust. The released latent heat would increase the temperature of the region by around $45 \mathrm{~K}$, and this temperature change could trigger changes in surrounding and deeper amorphous ice. The thermal pulse will move downwards into the nucleus and the phase change will continue to occur until the pulse reaches ice that is too cold to be heated above the transition temperature (140 K) by the latent energy release. Jewitt (1990) calculated that the internal temperature of $\mathrm{P} /$ Schwassmann-Wachmann $\mathrm{I}$ was around $110 \mathrm{~K}$. So if this comet had a water ice composition such that the upper $50 \mathrm{~cm}$ of the nucleus was crystalline and the remainder was amorphous, the normal coma activity would thin the crystalline sheath sufficiently to produce a few outbursts per year. Patashnick et al. found that $5 \times 10^{13} \mathrm{~g}$ of amorphous ice, transformed into cubic ice, would produce sufficient energy for an outburst (i.e., $10^{21} \mathrm{erg}$ ) and this could lead to the ejection of $10^{12} \mathrm{~g}$ of material from the nucleus. Obviously, if all the ice in the nucleus was amorphous and hotter than around $120 \mathrm{~K}$, the whole nucleus would disintegrate when the subsurface ice reached a temperature of $140 \mathrm{~K}$, with the release of latent heat causing a chain reaction. Fortunately, the presence of impurities inhibits the production of amorphous ice. Small pockets of amorphous ice, however, are all that are required. (Needless to say, you have to regard $5 \times 10^{13} \mathrm{~g}$ as small!)

This mechanism, like the two mentioned above, suffers by being temperature-dependent, and thus dependent on heliocentric distance. Comets have to have subsurface temperatures greater than $140 \mathrm{~K}$ for the mechanism to work, and this could be difficult in the case of, for example, $\mathrm{P} /$ Schwassmann-Wachmann I when it is close to its aphelion at around 7.4 AU. A more insurmountable objection arises from the fact that amorphous ice has only been produced in Earth laboratories under conditions of high purity and cleanliness. The dusty environment in which comets are born seems to be an unlikely place for the production of this ice. Also, the time scale for the conversion of amorphous to crystalline ice takes from minutes to hours in the laboratory (Sanford and Allamandola 1988), so the transition might not be 'explosive'. Prialnik and Bar-Nun (1987) concluded that the phase change would probably disrupt the whole nucleus, making it very difficult both for the comet to return to its original magnitude after outburst and also for successive outbursts to occur in the same nucleus.

There is another problem with the amorphous-ice theory. Most short-period comets have orbits that make them spend a large majority of their orbital period closer than $6 \mathrm{AU}$ to the Sun. These comets will have central nucleus temperatures that are higher than $140 \mathrm{~K}$ and so they will contain no amorphous ice.

\subsection{Impact Cratering By Interplanetary Boulders}

Sekanina (1972) investigated the possibility that the outbursts of $\mathrm{P} /$ SchwassmannWachmann I were caused by the impact of interplanetary boulders with the cometary nucleus. These boulders, rather like the asteroids, were thought to have direct orbits, and Sekanina suggested that they had a random distribution of perihelion distances in the 4.5to 7.5-AU range. (The near-uniform spatial density of large meteoroids in this region of 
the solar system has recently been suggested by the findings of Hughes and McBride 1990). Each collision produced an impact crater on the surface of the nucleus, and the cratering process caused both the ejection of dust and dirty snow and the exposure of a considerable area of underlying snow. These exposed regions and the ejected dirty snow then sublimated rapidly. In the region between 4.5 and $7.2 \mathrm{AU}$, the average impact velocity would be around $3 \mathrm{~km} \mathrm{sec}-1$.

The energy of a typical outburst is of the order of $10^{18}$ to $10^{19}$ ergs. An impacting boulder of mass $10^{8} \mathrm{~g}$ would have a similar kinetic energy. The observed expansion velocity of the dust seen during an outburst is of the same order of magnitude as that expected for the ejecta produced during an impact cratering event. The amount of mass released would be between 100 and 1000 times the mass of the impacting boulder. The change induced in the comet's orbit by the impact would be too small to be observable.

Some of the problems associated with this mechanism are as follows. First, there is only one point of impact on the comet's surface, and all the ejecta will emanate from that one spot. Unless the nucleus is spinning very rapidly, it is difficult to explain the symmetrical expanding dust clouds that have been seen in the majority of cases. This symmetry is, however, not universal, and a bright jet was seen issuing from the nucleus of $\mathrm{P} /$ TuttleGiacobini-Kresák after the peak of its outburst in 1973 . The $10^{8}-\mathrm{g}$ boulders would merely be the most effective members of a group of objects with a whole range of masses. More massive objects would be too rare to produce significant numbers of outbursts; the less massive boulders would be more plentiful, but would produce only minor outbursts, and these would have lower energies and would simply contribute to the general noise of the cometary brightness signal.

There is no fundamental reason why the $4.5<r<7.5 \mathrm{AU}$ region of the solar system should not contain a belt of orbiting boulders. The total surface area of the boulders in the suggested belt is so low that the amount of sunlight scattered by them would be much too faint to be detected from Earth. The boulders could be the remnant of an outer asteroid belt, a comet forming process, or simply large stream meteoroids near their aphelia. As such, their direct, low-inclination orbits would be no surprise, and this could explain why many near-parabolic comets (which have random orbital inclination) do not suffer outbursts as they pass through this region. A boulder impact mechanism is essentially random and would lead to a Poissonian distribution for the time intervals between outbursts.

Sekanina (1972) also emphasised that P/Schwassmann-Wachmann I underwent intermittent low-level activity during which the brightness fluctuated by 1 to 2 magnitudes for periods of a few months. He proposed that this was caused by the impact site remaining as a hot spot for a considerable time with the slow dissipation of this heat leading in part to snow sublimation.

The impact of boulders must occur and must lead to outbursts. But there is no reason why this should be the only responsible mechanism.

\subsection{Splitting}

Outbursts are often regarded as being minor forms of cometary breakups. Cometary splitting has been reviewed by Sekanina (1982). He noted that 21 comets have been observed to split, and that splitting occurred randomly with respect to heliocentric distance. Longperiod comets have a 1 in 25 chance of splitting per orbital revolution, and short-period 
ones have a 1 in 170 chance per orbit. The split portions separate gently, and their initial relative velocities are less than a few metres per second. In several cases, the initial breakup "coincides either with a flare-up in the visual and/or infrared brightness or with an outburst detected as an isolated streamer in the dust tail". The breadths of these streamers indicate that the duration of the dust bursts from the splitting comet lasted for not more than about 0.1 days. The duration of the brightness flare-up was usually much longer. The splitting of West (1976 VI) produced both a brightness flare and a substantial short-term increase in the dust emission. These were accompanied by about a dozen outbursts in the 2.5 weeks around perihelion. Comet Whipple-Fedtke-Tevzadze (1943 I) had two huge brightness outbursts that peaked 7 and 3 weeks before it broke apart (see Bayer 1948).

The lightcurves of the fragments that have split away from the main cometary body exhibit profound fluctuations, and the amplitude of these fluctuations indicate intensity variations of the order of 20:1 (i.e., about 3 magnitudes). The time intervals between maxima are of the order of 10 to 20 days. It seems likely that the fresh surface area revealed by the breakup produces a substantial gas and dust emission and represents a large percentage of the total active area of the fragment. The time interval between the maxima probably represents a rather complex combination of the rotation and precession period.

The mechanisms responsible for breakup are far from clear. Unfortunately the tensile strength ( $\sigma$ dynes $\mathrm{cm}^{-2}$ ) of cometary material is not well-known. Also, we need to recognise the possible mechanisms that can exert sufficient stresses on the nucleus. Tidal forces are one. Whipple (1963) showed that a comet of density $\rho$, tensile strength $\sigma$ and radius $R$ would be broken apart if it passed within a distance $D$ of a body of mass $M$ if

$$
\sigma<\frac{G M \rho R^{2}}{D^{3}}
$$

Excessive rotation can also lead to breakup. Disruption occurs if

$$
\sigma<\frac{2 \pi^{2} \rho R^{2}}{P_{c}^{2}}
$$

Particles at the nucleus equator will be moving at the escape velocity when the rotation period, $P_{c}$, is given by

$$
P_{\mathrm{c}}=3.30 \rho^{-0.5} \mathrm{hr} \text {. }
$$

So the higher the density of the object, the faster it can spin without splitting up. Analysis of cometary spin and sizes statistics (see Whipple 1982) leads to an upper limit for the tensile strength in the range $10^{3}$ to $10^{5}$ dynes $\mathrm{cm}^{-2}$. Even the upper of these two values is very low in comparison to the values for normal terrestrial materials.

Considering the low values now accepted for cometary densities, it can be concluded that all comets of densities near $0.25 \mathrm{~g} \mathrm{~cm}^{-3}$ and spin periods less than about two hours are in danger of splitting. The lowest spin period recorded by Whipple (1982) was a value of $4.14 \mathrm{hr}$, for comet Honda $(1955 \mathrm{~V})$. This comet did split, so the density of this comet must have been larger than the value given above. There is a tendency to regard objects of low tensile strength and low density as being somewhat porous.

Whipple and Stefanik (1966) suggested a splitting mechanism for the 'new' long-period comets that are probably entering the inner solar system for the first time. They suggested that these comets had suffered from internal radiogenic heating and this had caused the 
more volatile components of the interior snows to sublimate and then migrate towards the surface, where they condensed, forming a brittle shell. As the comet approached the Sun, the heat shock and the associated differential expansion exerted on this shell caused it to break up.

\subsection{Impacts By Companions}

Whipple (1985) investigated the possible existence of double comets, with these being both pairs of comets with nearly identical orbits and comets with attendant small satellite companions; du Toit-Hartley, P/Holmes, P/Neujmin 3, and P/van Biesbroeck were mentioned. If the two components suffer different non-gravitational jet forces, then their mutual orbits can change and a collision can result. Whipple found that two collisions probably occurred in the case of $\mathrm{P} / \mathrm{Holmes}$, with the first being a grazing encounter and the second, 73 days later, being a more 'head-on' blow and an impact that resulted in the destruction of the small satellite comet. After the first impact, the nucleus exhibited only one area of major activity, with a second one appearing after the second impact. Turning to P/Tuttle-Giacobini-Kresák, Whipple suggested that "it too may have been a double comet in which the larger of the twins cannibalised its sibling".

\subsection{Violent Crustal Breakup}

Sekanina (1982) regarded the secondary nuclei, which are released when a comet splits, as nothing more than fragments of the insulating mantle of the nucleus. These are 'shaken' free from the nucleus by tides or excessive spin speeds. As they move away, a large area of the layer of dirty snow that was at their base becomes exposed to sunlight, and the sublimation of this layer accounts for the large, but short-lived, luminosity outburst.

Note that Sekanina (1982) suggested that cometary outbursts were produced when a 'small' fragment of the crust was released. How large a portion of insulating crust can be built up on the surface of a comet? P/Halley has only a few percent of the surface active at any one time. Modelling the heat flow through cometary dust indicates that the dust layer over an active region has a thickness of a few millimetres at most. Inactive regions are covered with a thicker layer of dust. But how much thicker? A layer a few centimetres thick would provide a very effective insulator and would effectively block the sublimation of the underlying snow. Thus the thickness of this 'inactive' layer could not be significantly increased by the retreat of the underlying snows. Also, the thickness of this 'inactive' layer could not be decreased by the blow-off of surface dust by gas pressure. The region stagnates. Whipple and Sekanina (1979), however, suggested that things could get worse. They envisaged a scenario in which the inactive regions of the comet were being coated by large-sized dust that had been released from adjacent active areas, had failed to escape from the gravitational clutches of the comet and had then fallen back to the nucleus. As this process continued, the inactive region became thicker and thicker and the overlying weight of the accreted material led to a general compression and strengthening of the underlying dirty snow. Eventually this redistribution of mass began to affect the comet's rotation. The spinning non-spherical comet began to wobble more and more violently. Stresses built up, and from time to time these overcame the low tensile strength of the weaker regions of the nucleus. Note that we have no reason to expect the dirty snow mixture in the 
nucleus to be completely homogeneous and therefore it should contain some regions that are weaker than others. Spin-induced forces then caused a large region of crust, together with a reasonable section of the underlying dirty snow, to become detached. This detached surface could break up as it was released. The degree of disintegration and the degree of snow sublimation could account for a whole range of observed outburst characteristics and magnitudes.

Needless to say, there are problems. If large dust aggregates cannot escape and these then fall back to produce a thick mantle, why should very large sections of this mantle fare any better? The jet effect produced by the snow near the break point is very important in this context. This is illustrated by the fact that fragments from comets that split, break away with velocities, $V$, that are in the low metres per second $\left(V=0.7 r^{-0.57} \mathrm{~m} \mathrm{sec}{ }^{-1}\right.$; see Sekanina 1982). After release, the heliocentric velocity of the fragment usually becomes lower than that of the parent comet.

Comets must have some mechanism for ridding themselves of large sections of insulating 'inactive' crusts. Without these, the active areas would remain fixed in position and they would retreated further and further into the nucleus. Shadowing would increase and the active percentage of the surface would decrease and the topography of the comet would get more and more 'pitted'. The relative smoothness of the nucleus of $\mathrm{P} / \mathrm{Halley}$ indicates that the latter does not happen. So the positions of the active regions must change as a function of time. Hughes (1988b) found that the percentage of Halley's surface that was active seemed to vary randomly about an average value. The mean active percentages at $1 \mathrm{AU}$, for the returns in $1835,1759,1682,1607,1531$, and 1456 , were $3.9,4.3,0.9,5.6,2.2$ and 2.0 , respectively. So it can be seen that the surface of the nucleus can change significantly between apparitions. Comet $\mathrm{P} /$ Halley seems to be self-regulating. The surface is such that it opposes tendencies to either switch off the sublimation or to remove excessive sections of the overlying crust and thus introduce excessive activity and mass loss.

Thermal stresses might have a role to play in the breakup of the cometary surface. The temperature of the nucleus is cycled each orbit and this could easily lead to the cracking and loosening of the surface and subsurface regions simply due to the differential expansion of the snows and dust (see Kürht and Mohlmann 1984, and Tauber and Kürht 1987).

\subsection{Nuclear Crushing}

Kresák (1974) suggested that the depletion of volatiles in the deeper regions of the nucleus could lead to crustal shrinking and cave-ins. This could happen spasmodically, leading to the disintegration of regions of overlying insulating crust, the exposure of fresh snow, and the subsequent emission of gas and dust emission and an outburst in brightness.

\subsection{Solar Wind Triggering}

Neidner (1980) suggested that reactive species could be synthesised in cometary snows by long-term exposure to cosmic rays while the comet was part of the Oort-Öpik cloud complex. These might explode when exposed to high-energy (keV) solar wind protons, and these explosions will then be seen as outbursts.

This mechanism might account for outbursts on the pre-perihelion patl of long-period comets, but short-period comets lose their surface material so quickly that the exposure 
of this surface to cosmic rays is negligible. Also, the gas production in the vicinity of perihelion for a low $q$ comet is of the order of $10^{30} \mathrm{~mol} \mathrm{sec}^{-1}$, and this produces such a high ion density in the inner coma that the surface of the nucleus is effectively shielded from the incident solar wind.

\subsection{The Swiss Cheese Model}

I would like to take this opportunity to introduce a new mechanism for cometary outbursts, one that depends on physical and/or chemical inhomogeneities inside the cometary nucleus. One of the main problems of modern cometary physics is that we have very little knowledge of the internal structure of a cometary nucleus. This has not stopped many of us from indulging in the luxury of drawing cross-sections of nuclei (see, for example, Hughes 1975 and 1986a, Weissman 1986, and Wood 1986). The comets that we observe lose a considerable amount of mass at each perihelion passage. So the present-day surface of the nucleus of a short-period comet is completely different from the original surface of the nucleus. In fact, there is every possibility that the nucleus of $\mathrm{P} / \mathrm{Halley}$ was initially twice the size that it is now, and that the surface imaged by the Giotto spacecraft was initially halfway in towards the centre (see Hughes 1985 and 1987).

The cold accretion process responsible for cometary formation and the limited subsequent internal heating probably lead to a non-uniform internal density. The nucleus is made up of a fragile agglomeration of many smaller nuclei, with these having a vast range of sizes. Cometary nuclei thus have a fractal nature (see Daniel and Hughes 1981, and Donn and Hughes 1986). So the nucleus probably has regions of much reduced density, regions of different strengths and gas-to-dust ratios, and even holes where there is no material at all. The steady erosion of the surface of the comet, due to near-perihelion activity, could reveal one of these holes, suddenly exposing a considerable area of fresh snow to the solar radiation and thus leading to an outburst of activity. The establishment of a dust crust inside the hole cuts off this new activity and the comet then returns to normal (see Fig. 7).

A physical process that relies on there being a considerable range of internal densities could be augmented by a chemical inhomogeneity. The cometary snows could have a range of, say, $\mathrm{H}_{2} \mathrm{O} / \mathrm{CO}_{2}$ mass ratios. An outburst could then be triggered by the retreating surface revealing a region of high $\mathrm{CO}_{2}$ concentration at a time when the comet was in the inner solar system, and when the majority of the sublimation is governed by $\mathrm{H}_{2} \mathrm{O}$ sublimation. The comet quickly returns to normal when a more usual $\mathrm{H}_{2} \mathrm{O} / \mathrm{CO}_{2}$ ratio is encountered.

Note that the pressure at a distance $d$ from the centre of a comet of radius $R$ and density $\rho$ is given by

$$
P(r)=0.67 G \rho^{2}\left(R^{2}-d^{2}\right)
$$

During the lifetime of the comet, this pressure is always decreasing, so a hole that was present just after the accretion process has little tendency to disappear.

\section{Conclusions}

Two major findings have affected the list of acceptable outburst mechanisms. The first concerns the low density of cometary nuclei (typically $0.2 \mathrm{~g} \mathrm{~cm}^{-3}$ ) and the low tensile 
strength (typically $10^{4}$ dynes $\mathrm{cm}^{-2}$ ) of the cometary material. These values tend to rule out mechanisms that rely on the pressure build-up around pockets of gas. The nucleus is not solid, but is fragile and vesicular. Much of the interior contains voids. The gas would simply percolate out. Gas pressures are always negligible. The generation of forces by the sudden expansion of ice during a phase change also would be ineffective. This expansion would be akin to hammering a sponge.

Most exotic chemical mechanisms seem to be ruled out by their temperature sensitivity. The outburst occurrence rate would peak at certain heliocentric distances as the cometary nucleus changed temperature. Also, pre-perihelion locations, as opposed to post-perihelion ones, would be favoured. Neither of these characteristics is observed.

The second finding indicates that most comets are only active over a small percentage of the nucleus surface. Prior to the observations of $\mathrm{P} / \mathrm{Halley}$, most researchers thought that $50 \%$ to $100 \%$ of the nucleus was active. If this active percentage is of the order of $1 \%$, any mechanism that can change this to, for example, $2 \%, 4 \%, 8 \%, 16 \%$, or $32 \%$, for a short time, can increase the gas and dust emission by this ratio and can thus lead to an outburst of magnitude $0.8,1.5,2.3,3.0$ or 3.8. The magnitude change would be even larger

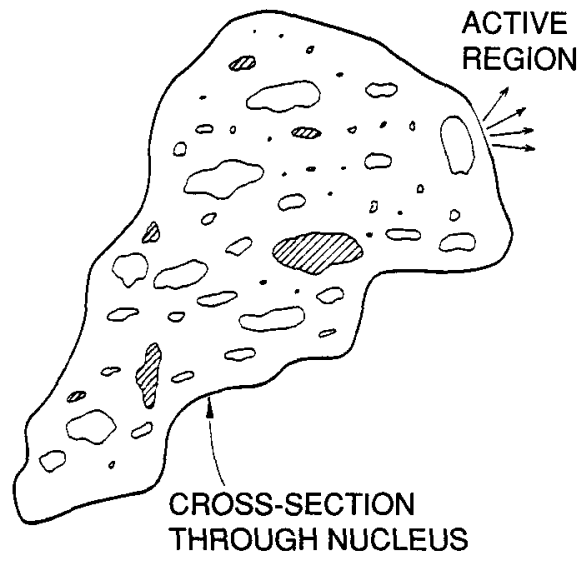

a)

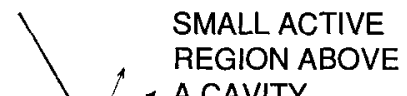

b)

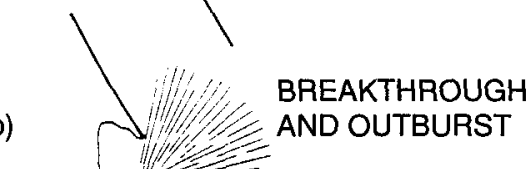

c)

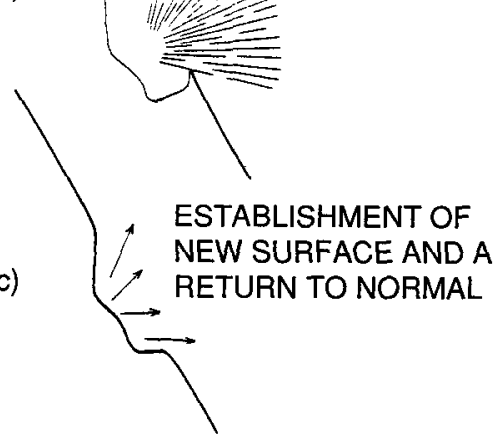

Fig. 7. This schematic diagram of a cross-section of a cometary nucleus shows two forms of interior inhomogeneity, with the open regions representing holes (which could be voids that have not been filled during the accretion process), and the hatched regions, representing volumes (planetesimals?) where the concentration of a more volatile species is higher than normal. As the surface of an active region retreats, it breaks through to one of these regions from time to time, and the fresh snow and the volatiles that are then exposed lead to an outburst. This additional activity ceases when the fresh snow and the volatiles are consumed. 
if dust fragmentation occurred during the process, simply because the area-to-mass-ratio of the dust would increase and even more light would be scattered. As the cometocentric velocity of the dust is proportional to about (mass) $)^{-1 / 6}$, the smaller the dust, the faster the cloud of scattering material is produced and the faster it dissipates. The section of the cometary surface that is released could break up soon after 'launch', at a time when the interstitial snows sublimate. Maybe it is these snows that are responsible for the majority of the strength of the material.

The removal of sections of the surface could be caused by the enhanced spin and precession of the nucleus. The variation in spin is not expected to take place gradually and some limit could have been exceeded just prior to the outburst. The majority of the change will probably occur at the times of maximum mass loss (i.e., just after perihelion passage).

Another mechanism relies on an external trigger, the collision between the comet and a 'boulder' of mass of the order of $10^{8} \mathrm{~g}$. These 'boulders' are not uncommon, so impacts between boulders and the surfaces of cometary nuclei will be reasonably frequent. The task of accelerating material away from the surface of the comet is not onerous; $\mathrm{P} / \mathrm{Halley}$ has an escape velocity of about $2 \mathrm{~m} \mathrm{sec}^{-1}$, and, as most comets are less massive, they will have even smaller escape velocities.

The phenomenological characteristics of the outbursts caused by the two mechanisms mentioned above will be indistinguishable. Both probably can, and do, occur. All an outburst needs is a source of energy (i.e., the kinetic energy of the incident boulder plus the absorbed radiation energy from the Sun), with this energy being applied to an ample supply of fresh cometary snow and fine dust.

We conclude that all comets can have outbursts. Whether the comet is observed to outburst depends solely on the percentage of the surface that was initially active. Imagine that the outburst mechanism introduces a new active region that is equivalent to gas and dust loss from, say, $2 \%$ of the surface. The effect of this temporary change on the brightness of the comet will depend to a very large extent on how much of the comet's surface was initially active. If it was, say, $0.5 \%$, the addition of a further $2 \%$ will change the brightness by a factor of 5 and the magnitude will change by 1.8 ; an outburst will be observed. If initially $20 \%$ of the surface was active, the addition of a further $2 \%$ will change the magnitude by a mere 0.1 and the outburst will be lost in the noise.

So outbursts are not rare; in fact, they are ubiquitous amongst the cometary community. All comets can and do suffer from outbursts. Many outbursts, however, are not detected because they are simply lost in the brightness noise. The only outbursts that are noticeable are those that occur on comets that initially had only a small region of the nucleus actively emitting gas and dust.

\section{References}

Beyer, M. (1948). 'Physische Beobachtungen von Kometan. VI.' Astron. Nachr., 275, $237-250$.

Brandt, J.C., and Mendis, D.A. (1979). 'The interactions of the solar wind with comets.' In Solar System Plasma Physics, Vol. II, C.F. Kennel, L.J. Lanzerotti, and E.N. Parker (eds.), North Holland Publ. Co., Amsterdam, pp. 253-292.

Campins, H., and Ferrin, I. (1978). 'Cometary outbursts.' Bull. Am. Astr. Soc., 10, 613. 
Cochran, A.L., Barker, E.S., and Cochran, W.D. (1980). 'Spectrophotometric observations of P/Schwassmann-Wachmann I during outburst.' Astron. J., 85, 474-477.

Cruikshank, D'P., and Brown, R.H. (1983). 'The nucleus of P/Schwassmann-Wachmann I.' Icarus, 56, 377-380.

Daniels, P.A., and Hughes, D.W. (1981). 'The accretion of cosmic dust - a computer simulation.' Mon. Not. R. Astr. Soc., 195, 1001-1009.

Degewij, J., and Tedesco, E.F. (1982). 'Do comets evolve into asteroids? Evidence from physical studies.' In Comets, L.L. Wilkening (ed.), University of Arizona Press, Tucson, pp. 85-130.

Delsemme, A.H. (1966). 'Vers un modele physico-chimique du noyau cometaire.' In Nature et Origine des Cometes, Mem. Soc. Roy. Sci. Liege, Ser. 5, 12, Universite de Liege, pp. $77-110$.

Delsemme, A.H. (1985). 'The sublimation temperature of the cometary nucleus: Observational evidence for $\mathrm{H}_{2} \mathrm{O}$ snows.' In Ices in the Solar System, J. Klinger, D. Benest, A. Dollfus, and R. Smoluchowski (eds.), D. Reidel Publ. Co., Dordrecht, pp. 367-387. Delsemme, A.H. (1986). 'Elemental isotopic and moelcular abundances in comets.' In Asteroids, Comets, Meteors II, C.-I. Lagerkvist, B.A. Lindblad, H. Lundstedt, and H. Rickman (eds.), Uppsala Universitet Reprocentralen, pp. 245-257.

Delsemme, A.H., and Wenger, A. (1970). 'Superdense water ices.' Science, 167, 44-45.

Donn, B., and Hughes, D.W. (1986). ' A fractal model of a cometary nucleus formed by random accretion.' In Exploration of Comet Halley, ESA SP-250, pp. 523-524.

Donn, B., and Urey, H.C. (1956). 'On the mechanism of cometary outbursts and the chemical composition of comets.' Astrophys. J., 123, 339-342.

Feldman, P.D., Festou, M.C., A'Hearn, M.F., Arpigny, C., Butterworth, P.S., Cosmovici, C.B., Danks, A.C., Gilmozzi, R., Jackson, W.M., McFadden, L.A., Patriachi, P., Schleicher, D.G., Tozzi, G.P., Wallis, M.K., Weaver, H.A., and Woods, T.N. (1986a). 'IUE observations of comet Halley: Evolution of the UV spectrum between September 1985 and July 1986.' In Exploration of Comet Halley, ESA SP-250, pp. 325-328.

Feldman, P.D., A'Hearn, M.F., Festou, M.C., McFadden, L.A., Weaver, H.A., and Woods, T.N. (1986b). 'Is $\mathrm{CO}_{2}$ responsible for the outbursts of comet Halley?' Nature, 324, $433-436$.

Flammer, K.R., Jackson, B., and Mendis, D.A. (1986). 'On the brightness variations of comet Halley at large heliocentric distances.' Earth, Moon and Planets, 35, 203-212.

Greenberg, J.M. (1986). 'Fluffy comets.' In Asteroids, Comets, Meteors II, C.-I. Lagerkvist, B.A. Lindblad, H. Lundstedt, and H. Rickman (eds.), Uppsala Universitet Reprocentralen, pp. 221-223.

Herman, G., and Weissman, P.R. (1987). 'Numerical simulation of cometary nuclei.' Icarus, 69, 314-328.

Horanyi, M., Gombosi, Y.I., Cravens, T.E., Korosmezey, K., Kecskemety, A.F., Nagy, A.F., and Szego, K. (1984). 'The friable sponge model of a cometary nucleus.' Astrophys. J., 278, 449-455.

Hughes, D.W. (1975). 'Cometary outbursts: A brief survey.' Quart. Jl. Roy. Astr. Soc., $16,410-427$.

Hughes, D.W. (1978). 'Meteors.' In Cosmic Dust, J.A.M. McDonnell (ed.), John Wiley and Sons, Chichester, pp. 123-185. 
Hughes, D.W. (1983). 'Cometary dust, its source and characteristics.' In Asteroids, Comets, Meteors I, C.-I. Lagerkvist and H. Rickman (eds.), Uppsala Universitet Reprocentralen, pp. 239-257.

Hughes, D.W. (1985). 'The size, mass, mass loss and age of Halley's comet.' Mon. Not. R. Astr. Soc., 213, 103-109.

Hughes, D.W. (1986a). 'Meteoroids: The best clues to the structure of a cometary nucleus?' In Comet Nucleus Sample Return, ESA SP-249, pp. 173-179.

Hughes, D.W. (1986b). 'The relationship between comets and meteoroid streams.' In Asteroids, Comets, Meteors II, C.-I. Lagerkvist, B.A. Lindblad, H. Lundstedt, and H. Rickman (eds.), Uppsala Universitet Reprocentralen, pp. 503-519.

Hughes, D.W. (1987). 'The history of Halley's comet.' Phil. Trans. R. Soc. Lond., A323, 349-367.

Hughes, D.W. (1988a). 'Cometary magnitude distribution and the relationship between the number of short- and long-period comets.' Icarus, 73, 149-162.

Hughes, D.W. (1988b). 'The brightness of P/Halley and the surface activity of the nucleus at previous apparitions.' Mon. Not. R. Astr. Soc., 234, 173-176.

Hughes, D.W., and McBride, N. (1990). 'The spatial density of large meteoroids in the inner solar system.' Mon. Not. R. Astr. Soc., in press.

Hughes, D.W., Searle, R.M., and Street, R.A. (1974). 'Spatial distribution of cometary outbursts.' Nature, 253, 615-616.

Jewitt, D. (1990). 'The persistent coma of comet P/Schwassmann-Wachmann I.' Astrophys. J., in press.

Keller, H.U., Delamere, W.A., Huebner, W.F., Wilhelm, K., Curdt, W., Kramm, R., Thomas, N., Arpigny, C., Barbiera, C., Bonnet, R.M., Case, S., Coradini, M., Cosmovici, C.B., Hughes, D.W., Jamar, C., Malaise, D., Schmidt, K., Schmidt, W.K.H., and Seige, P. (1987). 'Comet P/Halley's nucleus and its activity.' Astron. Astrophys., 187, 807-823.

Klinger, J. (1980). 'Influence of a phase transition of ice on the heat and mass balance of comets.' Science, 209, 271-272.

Krankowsky, D., Lämmerzahl, P., Herrwerth, I., Woweries, J., Eberhardt, P., Dolder, U., Herrmann, U., Schulte, W., Berthelier, J.J., Iliano, J.M., Hodges, R.R., and Hoffman, J.H. (1986). 'In situ gas and ion measurement at comet Halley.' Nature, 321, 326-329.

Kresák, L. (1966). 'On two aspects of the evolution of short-period comets.' In Nature et Origine des Cometes, Mem. Soc. Roy. Sci. Liege, Ser. 5, 12, Universite de Liege, pp. 459-480.

Kresák, L. (1974). 'The outbursts of periodic comet Tuttle-Giacobini-Kresák.' Bull. Astr. Inst. Czech., 25, 293-304.

Kührt, E., and Mohlmann, D. (1984). 'Thermal stress in cometary nuclei.' Gerl. Beitr. Geoph., 93, 232-234.

Larson, H.P., Hong-Yau, H., Mumma, M.J., and Weaver, H.A. (1989). 'Outburst of $\mathrm{H}_{2} \mathrm{O}$ in comet Halley.' Icarus, in press. 
McDonnell, J.A.M., Kissel, J., Grun, E., Grard, R.J.L., Langevin, Y., Olearczyk, R.E., Perry, C.H., and Zarnecki, J.C. (1986). 'Giotto's dust impact detection system DIDSY and the particulate impact analyser PIA: Interim assessment of the dust distribution and properties within the coma.' In Exploration of Comet Halley, ESA SP-250, Vol. II, pp. 25-38.

McDonnell, J.A.M., Pankiewics, G.S., Birchley, P.N.W., Green, S.F., and Perry, C.H. (1989). 'The in-situ cometary particulate size distribution measured for one comet: P/Halley.' In Proceedings of Workshop on Analysis of Returned Comet Nucleus Samples, Milpitas, California, in press.

Miller, S.L. (1961). 'The occurrence of gas hydrates in the solar system.' Proc. Nat. Acad. Sci., 47, 1798.

Niedner, M.B. (1980). 'Interplanetary gas XXV. A solar wind and interplanetary magnetic field interpretation of cometary light outbursts.' Astrophys. J., 241, 820-829.

Patashnick, H., Rupprecht, G., and Schuerman, D.W. (1974). 'Energy source for cometary outburst.' Nature, 250, 313-314.

Peale, S.J. (1989). 'On the density of Halley's comet.' Icarus, 82, 36-49.

Pittich, E.M. (1969). Bull. Astr. Inst. Czech., 20, 251.

Pittich, E.M. (1971). 'Space distribution of the splitting and outbursts of comets.' Bull. Astr. Inst. Czech., 22, 143-153.

Pittich, E.M. (1972). 'Splitting and sudden outbursts of comets as indicators of nongravitational effects.' In The Motion, Evolution of Orbits and Origin of Comets, I.A.U. Symposium 45, G.A. Chebotarev and E.I. Kazimirchak-Polonskaya (eds.), pp. 283286.

Prialnik, D., and Bar-Nun, A. (1987). 'On the evolution and activity of cometary nuclei.' Astrophys. J., 313, 893-905.

Richter, N.B. (1941). 'Helligkeitsschwankungander kometen und sonnentatigkeit: I. Erster Schwassmann-Wachmann'scher komet (1925 II).' Astr. Nachr., 271, 207.

Richter, N.B. (1948a). 'Helligkeitsschwankungen der kometen und sonnentatigkeit: II. Statistigk und theorie abnormer lichtausbruche von kometenkernen.' Astr. Nachr., $277,12$.

Richter, N.B. (1948b). 'Objekt keuskamp und das problem der unbestatigten kometenentdeckungen.' Stern, 24, 52.

Richter, N.B. (1954). 'Die helligkeitsausbruche des kometen 1925 II (SchwassmannWachmann I) und ihre korrelation zu erdmagnetischen ereignissen.' Astr. Nachr., $281,241$.

Richter, N.B. (1963). The Nature of Comets, Methuen, London, p. 142.

Rickman, H. (1986). 'Masses and densities of comets Halley and Kopff.' In Comet Nucleus Sample Return, ESA SP-249, pp. 195-205.

Rickman, H., Kamel, L., Festou, M.C., and Froeschle, C. (1987). 'Estimates of the mass, volume and densitites of short-period comet nuclei.' In Diversity and Similarity of Comets, ESA SP-278, pp. 471-481.

Roemer, E. (1958). 'An outburst of comet Schwassmann-Wachmann I.' Publ. Astron. Soc. Pacific, 70, 272.

Roemer, E. (1962). 'Activity in comets at large heliocentric distances.' Publ. Astron. Soc. Pacific, 74, 351-365. 
Roemer, E. (1963). 'Comets: Discovery, orbits, astrometric observation.' In The Moon, Meteorites, and Comets, B.M. Middlehurst and G.P. Kuiper (eds.), University of Chicago Press, Chicago, pp. 527-549.

Roemer, E., and Lloyd, R.E. (1966). 'Observations of comets, minor planets and satellites.' Astron. J., 71, 443-457.

Sandford, S.A., and Allamandola, L.J. (1988). 'The condensation and vapourization behavior of $\mathrm{H}_{2} \mathrm{O}: \mathrm{CO}_{2}$ ices and implications for interstellar grains and cometary activity.' Icarus, $76,210-224$.

Sekanina, Z. (1972). 'Outbursts of comet Schwassmann-Wachmann I, and the cloud of interplanetary boulders.' In Asteroids, Comets, Meteoric Matter, I.A.U. Colloquium 22, Nice, France, April 1972.

Sekanina, Z. (1982). 'The problem of split comets in review.' In Comets, L.L. Wilkening (ed.), University of Arizona Press, Tucson, pp. 251-287.

Smoluchowski, R. (1981). 'Amorphous ice and the behaviour of cometary nuclei.' Astrophys. J., 244, L31-L34.

Smoluchowski, R. (1985). 'Amorphous ice and porous ice in cometary nuclei.' In Ices in the Solar System, J. Klinger, D. Benest, A. Dollfus, and R. Smoluchowski (eds.), D. Reidel Publ. Co., Dordrecht, pp. 397-406.

Smoluchowski, R. (1986). 'Brightness curve and porosity of cometary nuclei.' In Asteroids, Comets, Meteors II, C.-I. Lagerkvist, B.A. Lindblad, H. Lundstedt, and H. Rickman (eds.), Uppsala Universitet Reprocentralen, pp. 305-315.

Tauber, F., and Kürht, E. (1987). 'Thermal stress in cometary nuclei.' Icarus, 69, 83-90.

Venkatesh, C.G., Rice, S.A., and Narten, A.H. (1974). 'Amorphous solid water: An X-ray diffraction study.' Science, 186, 927-928.

Vsekhsviatskii, S.K. (1966). 'Comet flares and the nature of the cometary nucleus.' In Nature et Origine des Cometes, Mem. Soc. Roy. Sci. Liege, Ser. 5, 12, Universite de Liege, pp. 53-64.

Walker, M.F. (1959). 'Observations of comet Schwassmann-Wachmann I during an outburst.' Publ. Astron. Soc. Pacific, 71, 169.

Wallis, M.K., and MacPherson, A.K. (1981). 'On the outgassing and jet thrust of snowball comets.' Astron. Astrophys., 98, 45-49.

Weissman, P.R. (1986). 'How pristine are cometary nuclei?' In Comet Nucleus Sample Return, ESA SP-249, pp. 15-25.

Whipple, F.L. (1951). 'A comet model I: The acceleration of comet Encke.' Astrophys. J., 111, 375-394.

Whipple, F.L. (1963). 'On the structure of the cometary nucleus.' In The Moon, Meteorites, and Comets, B.M. Middlehurst and G.P. Kuiper (eds.), University of Chicago Press, Chicago, pp. 639-664.

Whipple, F.L. (1980). 'Rotation and outbursts of comet P/Schwassmann-Wachmann I.' Astron. J., 85, 305-313.

Whipple, F.L. (1982). 'The rotation of cometary nuclei.' In Comets, L.L. Wilkening (ed.), University of Arizona Press, Tucson, pp. 227-250.

Whipple, F.L. (1985). The Mystery of Comets, Smithsonian Inst. Press, Washington, D.C., p. 173. 
Whipple, F.L. (1987). 'The cometary nucleus: Current concepts.' Astron. Astrophys., $187,852-858$.

Whipple, F.L.', and Sekanina, Z. (1979). 'Comet Encke: Precession of the spin axis, nongravitational motion and sublimation.' Astron. J., 84, 1894-1909.

Whipple, F.L., and Stefanik, R.P. (1966). 'On the physics and splitting of cometary nuclei.' In Nature et Origine des Cometes, Mem. Soc. Roy. Sci. Liege, Ser. 5, 12, Universite de Liege, pp. 33-52.

Whitney, C.A. (1955). 'Cometary outbursts.' Astrophys. J., 122, 190-195.

Wood, J.A. (1986). 'Cometary nucleus models: A review.' In Comet Nucleus Sample Return, ESA SP-249, pp. 123-131.

Yeomans, D.K. (1989). Icarus, in press. 